\title{
Effect of OM-85 BV on reducing bronchiectasis exacerbation in Chinese patients: the iPROBE study
}

\author{
Jinming Gao ${ }^{1}$, Lun $\mathrm{Li}^{1}$, Nan Jiang ${ }^{2}$, Ying $\mathrm{Liao}^{2}$, Lingfei Kong ${ }^{3}$, Yuanlin Song ${ }^{4}, \mathrm{Jinfu}_{\mathrm{Xu}}{ }^{5}, \mathrm{Jie} \mathrm{Cao}^{6}$, \\ Yuping $\mathrm{Li}^{7}$, Chengli Que ${ }^{8}$, Roy A. Pleasants'
}

${ }^{1}$ Department of Pulmonary and Critical Care Medicine, Peking Union Medical College Hospital, Peking Union Medical College \& Chinese Academy of Medical Sciences, Beijing, China; ${ }^{2}$ Department of Medicine, Peking Union Medical College \& Chinese Academy of Medical Sciences, Beijing, China; ${ }^{3}$ Department of Pulmonary and Critical Care Medicine, First Affiliated Hospital of China Medical University, Shenyang, China; ${ }^{4}$ Department of Pulmonary and Critical Care Medicine, Zhongshan Hospital, Fudan University, Shanghai, China; ${ }^{5}$ Department of Pulmonary and Critical Care Medicine, Shanghai Pulmonary Hospital, Tongji University, Shanghai, China; ${ }^{6}$ Department of Pulmonary and Critical Care Medicine, Tianjin Medical University General Hospital, Tianjin, China; ${ }^{7}$ Department of Pulmonary and Critical Care Medicine, First Affiliated Hospital of Wenzhou Medical University, Wenzhou, China; ${ }^{8}$ Department of Pulmonary and Critical Care Medicine, First Hospital of Peking University, Beijing, China; ${ }^{9}$ Division of Pulmonary Medicine and Critical Care, Chapel Hill, NC, USA

Contributions: (I) Conception and design: J Gao, RA Pleasants; (II) Administrative support: J Gao, L Kong, Y Song, J Xu, J Cao, Y Li, C Que; (III) Provision of study materials or patients: J Gao, L Kong, Y Song, J Xu, J Cao, Y Li, C Que; (IV) Collection and assembly of data: J Gao, L Li, N Jiang, Y Liao; (V) Data analysis and interpretation: J Gao, RA Pleasants; (VI) Manuscript writing: All authors; (VII) Final approval of manuscript: All authors.

Correspondence to: Jinming Gao, MD. Department of Pulmonary and Critical Care Medicine, Peking Union Medical College Hospital, \#1 Shuaifuyuan, Dongcheng District, Beijing 100730, China. Email: gjinming@yahoo.com.

Background: Bronchiectasis is characterized by recurrent infectious exacerbations. No existing data inform preventive strategy for exacerbations beyond chronic macrolides. OM-85 BV, an immunostimulant, has been shown to prevent recurrent respiratory infections. We initiated this 1-year, multi-centered, double-blind, and controlled trial to investigate the PReventive effect of OM-85 BV on Bronchiectasis Exacerbations in Chinese patients (iPROBE).

Methods: Patients with bronchiectasis aged 18 to 75 years, having at least one exacerbation in the past year, were randomized to receive, in addition to any respiratory medications, two courses of $7 \mathrm{mg}$ of OM-85 BV or matching placebo (one capsule orally per day for 10 days a month) for 3 consecutive months, followed by 3 months without treatment. The primary outcomes included the number of acute infectious exacerbations and the time to first exacerbation. Secondary endpoints included patient-reported respiratory outcomes. Safety measures were also assessed.

Results: Among the 196 participants, 99 were in the OM-85 BV group and 97 in the placebo group. At week 52, the mean number of acute exacerbations per patient was equal to 0.98 and 0.75 , respectively, in the two groups $(\mathrm{P}=0.14)$. Difference in the time to first pulmonary exacerbation was not statistically significant $(\mathrm{P}=0.11)$. There was no statistically significant difference in any secondary end-points. The safety profile in the two arms was good and the majority of adverse events were mild.

Conclusions: OM-85 BV did not demonstrate protection in decreasing pulmonary exacerbations of bronchiectasis in this trial performed in Chinese patients. It had good safety profile.

Keywords: OM-85 BV; bronchiectasis; immunostimulation; infection; pulmonary exacerbation

Submitted Apr 14, 2020. Accepted for publication Jan 22, 2021.

doi: $10.21037 /$ jtd-20-1662

View this article at: http://dx.doi.org/10.21037/jtd-20-1662 


\section{Introduction}

Non-cystic fibrosis bronchiectasis (thereafter called bronchiectasis) is a common, but, long-term neglected chronic respiratory disease in China $(1,2)$. Indeed, prevalence of bronchiectasis in adult populations has been rising significantly around the world $(3,4)$. Bronchiectasis represents a suppurative airway disorder with its phenotypes being highly heterogeneous and is characterized by permanent dilatation of one or more bronchi (5).

The abnormal architecture of bronchiectatic airways facilitates the chronic colonization of bacterial species such as Haemophilus influenzae, Streptococcus pneumoniae and Pseudomonas aeruginosa (6-8). Therefore, patients with bronchiectasis are prone to recurrent pulmonary exacerbations caused by bacterial infections, negatively influencing the natural history of the disease (9). Progressive airway damage and a deregulated immune response, resulting from a "vicious cycle" of recurrent bacterial infections, reflect a failure of the host defenses to maintain the homeostasis of the respiratory tract (7). Therefore, prevention of pulmonary exacerbations should be a prioritized aim of therapy. However, there is a limited evidence base to support the regimes for this goal (10).

Chronic macrolides with both antibacterial and antiinflammatory properties have shown promise in preventing bronchiectasis exacerbations (11). Six to 12 months of treatment with these medications has shown a decreased rate of bronchiectasis exacerbations by several randomized controlled trials (RCTs) and a pooled meta-analysis (12-15). Nevertheless, chronic use of macrolides significantly increased bacterial resistance to antibiotics as well as potential adverse drug effects (14-16).

The non-specific prevention of recurrent airway infections by immunostimulating agents has gained recognition over the past decades. OM-85 BV (Bronchovaxom, Vifor Pharma Pharmaceutics, Geneva, Switzerland), an immunostimulant, contains of extracts of eight different bacteria thought to be commonly responsible for respiratory infections. Orally administrated OM-85 BV increases respiratory immune defense against a wide variety of pathogens by activating pulmonary macrophages, increasing the CD4/CD8 lymphocytes ratio and IFN- $\gamma$ concentration in the human lung (17). OM-85 BV has been shown to effectively reduce exacerbations and improve life quality for patients with chronic obstructive pulmonary disease (COPD) in the RCTs (18-20).

Given that the majority of patients with bronchiectasis often suffer from bacterial exacerbations (21), we implemented this investigator-initiated and -driven, multicentered, phase 4 trial of investigation of PReventive effect of OM-85 on Bronchiectasis Exacerbations in adult Chinese patients (iPROBE).

\section{Methods}

\section{Study design and subjects}

Detailed design of this trial has been published previously (22). This study was a 52-week, randomized, double-blind, and placebo-controlled trial carried out in ten medical centers in Beijing, Tianjin, Shenyang, Shanghai, Hangzhou, and Wenzhou in China.

We enrolled bronchiectasis patients at routine outpatient visits at the time of clinical stability. Follow-ups were carried out in subspecialty clinics at study sites. Participants underwent study visits at the participating clinical centers on five separate occasions and had two follow-ups by telephone call during the 1-year trial period (Figure 1). The end of the study was defined by the last visit of the last participant. Tests performed during the visits were described previously (22).

The diagnosis of bronchiectasis was made as per the British Thoracic Society guidelines (21). Patients were eligible if they met the following inclusion criteria: subjects of both genders were adult patients ( $>18$ years) with bronchiectasis shown by chest high-resolution computed tomography (HRCT) within 3 years of study inclusion and having had at least one acute exacerbation in the previous 1 year when recruiting. An exacerbation was defined as the patient reporting that an acute worsening in signs and symptoms that needing additional therapy (23). Full exclusion criteria and withdrawal criteria were provided in the published protocol (22).

\section{Randomization and intervention}

All eligible subjects were randomized using block randomization sequences generated by computer. The subjects were assigned at a 1:1 ratio with a permuted block size of four and sequential assignment, stratified by the center. Blinding continued until all analyses were completed.

The subjects were randomized to receive two courses of $7 \mathrm{mg}$ of OM-85 BV or matching placebo (one oral capsule per day for 10 days a month) for 3 consecutive months, followed by 3 months without treatment. The trial lasted for 1 year (Figure 1). 


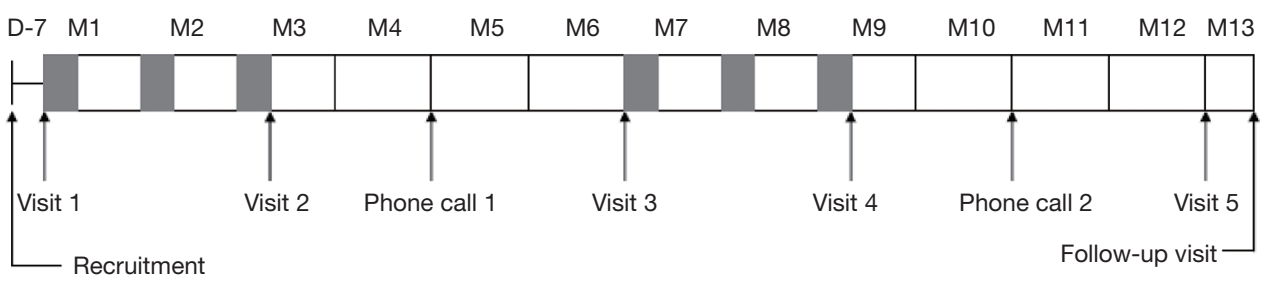

$\begin{array}{ll}\text { Treatment } & \text { No treatment }\end{array}$

Figure 1 Treatment periods and visits.

\section{Outcomes}

The primary goals of this study include two domains during 12-month period: (I) the number of event-based acute exacerbation; (II) time to first acute exacerbation.

The secondary efficacy variables included: life quality assessed by the St. George's Respiratory Questionnaire (SGRQ) (24); cough severity using Leicester Cough Questionnaire (LCQ) score (25); change in lung function parameters reflected by forced expiratory volume in one second (FEV1), forced vital capacity (FVC), FEV1/FVC; serum C-reactive protein (CRP); duration of antibiotics use; use of a rapid-acting bronchodilator; and bacteria clearance of spontaneously expectorated sputum.

\section{Sample size}

To detect a true difference in the primary outcome measures of number of bronchiectasis exacerbations and time to first exacerbation, a total of 244 subjects (122 per arm) were screened. This calculation was based on the $80 \%$ probability of detecting the statistically and clinically significant difference in the number of bronchiectasis exacerbations and time to the first exacerbation (22).

\section{Ethical approval}

The study was approved by the Human Research Ethics Board at Peking Union Medical College Hospital (REB number S-474) and was registered with www.clinicaltrials. gov (identifier number NCT01968421). Informed written consent was obtained from each participant.

The study was conducted in accordance with the Declaration of Helsinki (as revised in 2013), the International Conference for Harmonization (ICH) principles of Good Clinical Practice (GCP) and the Declaration of
Tokyo (2004). The investigators conducted all aspects of this study in accordance with all national and regional laws of the pertinent regulatory authorities.

\section{Statistics}

There were three analysis populations in the study: Full Analysis Set (FAS), Per Protocol Set (PPS) and Safety Set. The primary endpoints were analyzed on FAS, which was the primary population and supported by PPS. The secondary endpoint and baseline information were analyzed on FAS. Safety analyses were performed on Safety Set.

The primary efficacy variable was analyzed on FAS population and supported by PPS population. Statistical description and statistical inference were conducted. Mean \pm standard deviation (SD) was presented as a statistical description by study group.

Statistical inference consisted of parameter estimation and hypothesis test. For categorical variables, frequency and proportion were summarized by groups. Comparisons between two groups were performed using Student's $t$-test or Wilcoxon rank-sum test depending on data distribution. Two-tailed $95 \%$ confidence interval of the difference between groups was also presented.

The categorical data were analyzed by Pearson chisquare test or Fisher exact test. The change after and before treatment within each group was described and tested by paired $t$-test or signed ranks test.

The interval to the first time of acute exacerbation was analyzed as survival data. Kaplan-Meier curve of two groups was presented and log-rank test was used to test the difference between two groups.

All analyses followed the intention-to-treat principle. We employed the following methods to minimize the potential effects of missing data or dropout. First, when the study treatment was discontinued, we tried to obtain consent from 
the participants to collect data on treatments and outcomes. We used an estimating equation method to impute missing data in our primary analysis and we conducted a sensitivity analysis by using simple imputation methods (26). All reported $\mathrm{P}$ values are two-sided. All data analyses were performed using SAS 9.4 (SAS Institute Inc.).

\section{Safety analysis}

Analysis on safety was done using safety set. All adverse events (AEs) were coded using the version of MedDRA. Total frequency and incidence of $\mathrm{AE}$ and severe $\mathrm{AE}$ (SAE) were described. The frequency of AE in each group was tabulated by system organ class. The severity and relationship with the study were summarized by each group.

\section{Results}

\section{Study participants}

The first patient was enrolled March 1, 2015 and the last visit was on December 22, 2017. Between January 2, 2015 and January 9, 2018, a total of 244 clinically stable subjects with a diagnosis of bronchiectasis were recruited from ten medical centers (Table 1). Of these, 196 patients from ten study sites were randomized into OM-85 BV group ( $\mathrm{n}=99)$ or placebo group ( $\mathrm{n}=97)$, and $175(89.3 \%)$ completed the treatment and trial. All participants reported more than one exacerbation in the previous 1 year at recruitment. Figure 2 shows the reasons for trial withdrawal.

The two treatment arms were generally comparable in terms of sex, age, smoking status, length of bronchiectasisrelated symptoms, number of acute exacerbations in the past year, lung function parameters, and serum inflammatory markers (Table 1). The OM-85 BV group differed from the placebo group in more patients $(64.6 \%)$ with bronchiectasis for more than 10 years $(\mathrm{P}=0.02)$. The significantly higher percentage of patients using inhaled anticholinergics in OM-85 BV group compared with placebo group (37.2\% vs. $24.0 \%, \mathrm{P}=0.047)$. No chronic exposure to antibiotics was reported among subjects in the two groups.

In this trial, Pseudomonas aeruginosa was the most often isolated pathogen in sputum, seen in $32.9 \%$ in OM-85 BV group and $25.9 \%$ in placebo group. Group A Streptococcus in sputum was the secondary most common isolated bacteria, where it was present in $9.8 \%$ of patients in OM-85 BV group and $12.9 \%$ of patients in the placebo group.

\section{Efficacy}

\section{Primary outcomes}

Over the 1-year study period, the number of one or more episodes of acute exacerbation per patient per 6 months, did not differ between the two groups OM-85 BV or placebo ( $0.57 \pm 0.76$ vs. $0.42 \pm 0.66, \mathrm{P}=0.16$ ) (Table 2). At week 52, the mean rate of acute exacerbation episodes was equal to $0.98 \pm 1.19$ and $0.75 \pm 0.98$, respectively, in the two groups $(\mathrm{P}=0.14$ ) (Table 2).

Because there was no beneficial effect for OM-85 BV on bronchiectasis exacerbation, we next analyzed the impact of inhaled corticosteroids (ICS) on pulmonary exacerbations in OM-85 BV group. In the OM-85 BV group, $24.5 \%$ of patients had taken ICSs and found that patients never on ICSs had fewer, not statistically significant, exacerbations than that ever on ICSs at week $24(0.40 \pm 0.66 v s$. $0.73 \pm 0.70$, $\mathrm{P}=0.08)$, however, this effect was not shown at week 52 $(0.72 \pm 1.01$ vs. $1.00 \pm 0.78, \mathrm{P}=0.33)$ (Table 3).

Also, there was no difference in the time to first exacerbation after 1year between the two groups $(\mathrm{P}=0.11)$ (Figure 3). In a prior study, local airway immune response was modified by oral delivery of OM-85 BV after 3 months (27). We further assessed the difference in the time to the second flare-up between the two groups, the difference was not statistically significant (data not shown).

\section{Secondary outcomes}

At weeks 26 and 52, there were no statistically significant differences between OM-85 BV group and placebo group for any secondary efficacy outcomes including cough severity assessed by LCQ, life quality expressed by St. George scores, lung function measures, and serum inflammatory indices (Table 4). Likewise, there were no statistically significant differences in duration of acute exacerbation $(8.84 \pm 4.44$ vs. $9.11 \pm 4.15$ days, $\mathrm{P}=0.723)$ and days of antibiotics administration $(9.19 \pm 4.48$ vs. $9.20 \pm 3.82$ days, $\mathrm{P}=0.989)$ throughout the study course (Table 5). Also, the use of rapidacting bronchodilators as rescue medication did not differ in the two groups (data not shown).

\section{Safety analysis}

The safety profile and tolerability of OM-85 BV in this study were generally good as reported in previous studies performed in COPD exacerbation trials $(19,20,28)$. Sixteen AEs were reported totally (7 in OM-85 BV group and 9 in placebo group) (Table 6). Most AEs were mostly mild, 
Table 1 Patient demographics and baseline clinical characteristics

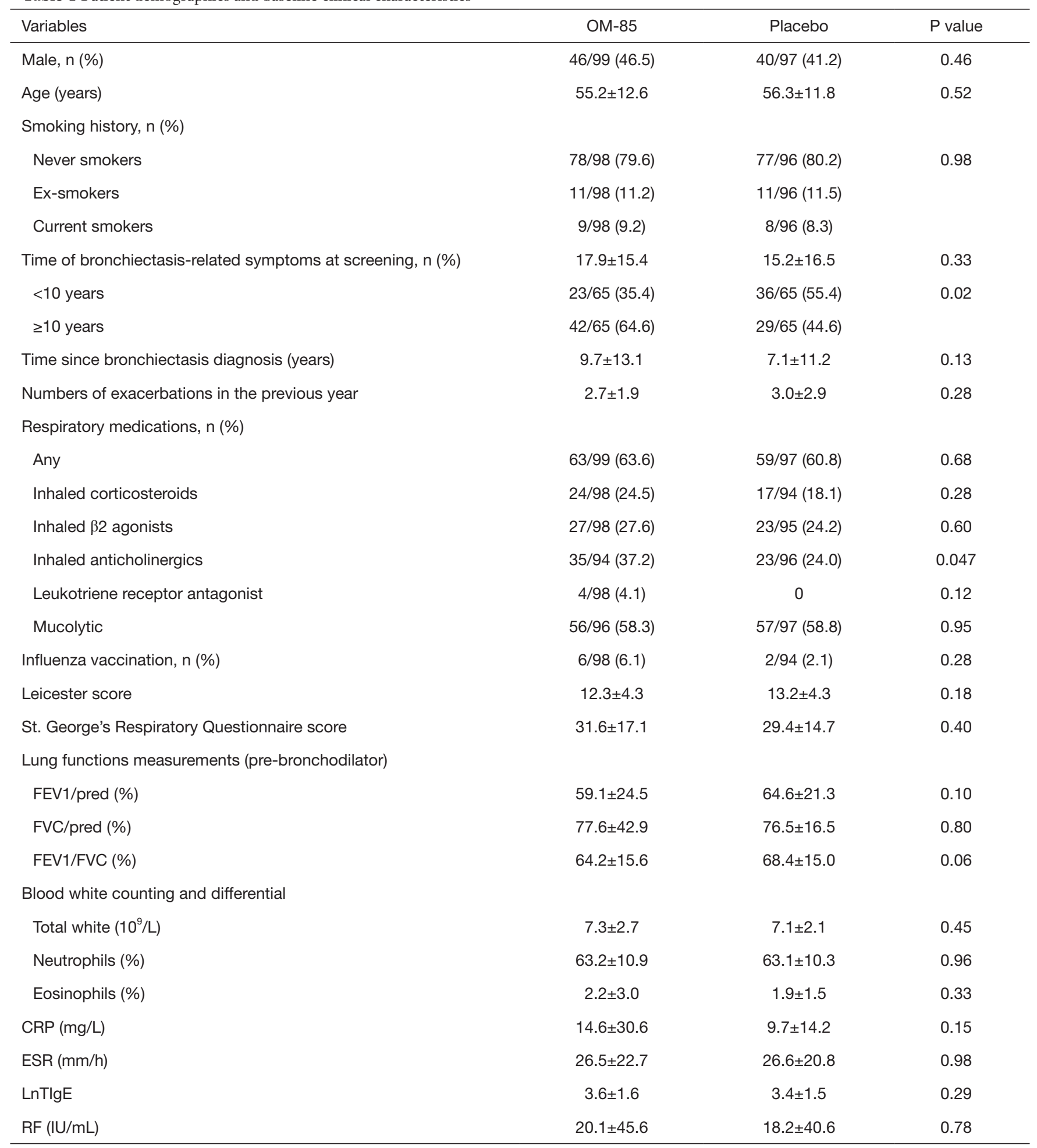

FEV1/pred, forced expiratory volume in first second/predicated; FVC/pred, forced vital capacity/predicated; CRP, C-reactive protein; ESR, erythrocyte sedimentation rate; RF, rheumatoid factor; LnTIgE, log-transformed IgE. 


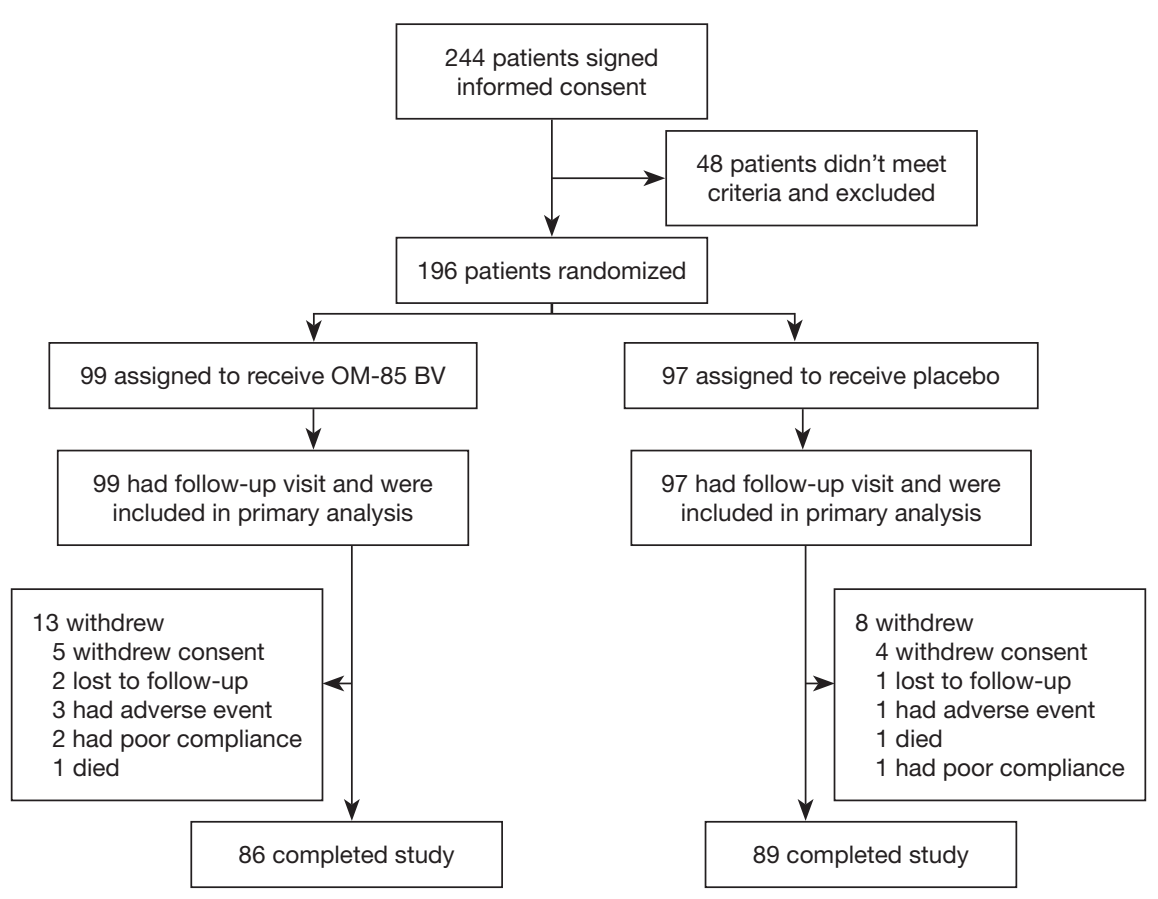

Figure 2 Study flow.

Table 2 Cumulative number of acute exacerbations per patient

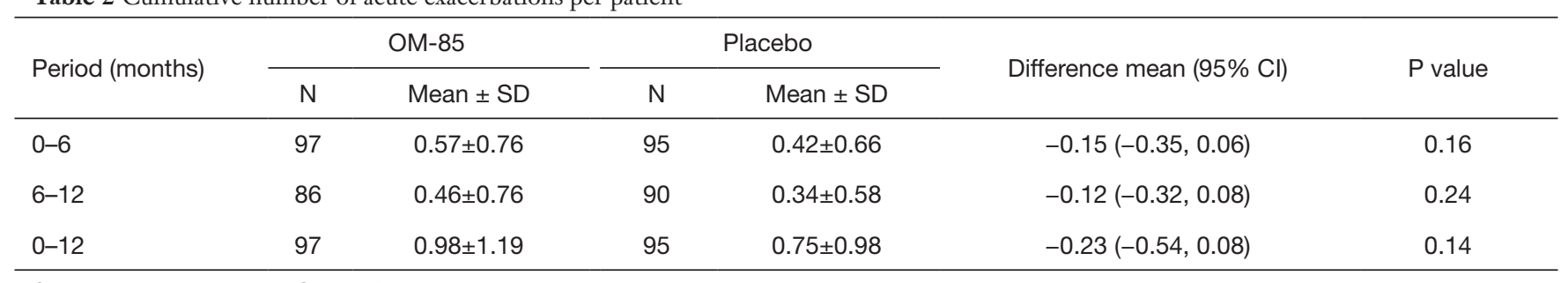

$\mathrm{SD}$, standard deviation; $\mathrm{Cl}$, confidence interval.

Table 3 Impact of ICSs on cumulative number of acute exacerbations per patient

\begin{tabular}{|c|c|c|c|c|c|c|}
\hline Period (months) & \multicolumn{2}{|c|}{ No ICSs } & \multicolumn{2}{|c|}{ ICSs } & Difference mean (95\% Cl) & $P$ value \\
\hline $0-6$ & 73 & $0.40 \pm 0.66$ & 15 & $0.73 \pm 0.70$ & $-0.33(-0.71,0.04)$ & 0.08 \\
\hline $6-12$ & 72 & $0.33 \pm 0.58$ & 14 & $0.36 \pm 0.63$ & $-0.03(-0.37,0.32)$ & 0.89 \\
\hline $0-12$ & 72 & $0.72 \pm 1.01$ & 14 & $1.00 \pm 0.78$ & $-0.28(-0.85,0.29)$ & 0.33 \\
\hline
\end{tabular}

ICS, inhaled corticosteroids; SD, standard deviation; $\mathrm{Cl}$, confidence interval.

including common cold, abdominal discomfort, nausea, and arthralgia. SAEs were observed in OM-85 BV group ( $\mathrm{n}=2)$ and placebo group ( $\mathrm{n}=1)$, no serious AEs were attributed to the medication and no significant laboratory changes were observed.

\section{Discussion}

This 52-week, randomized, double-blind trial evaluated the potential of $\mathrm{OM}-85 \mathrm{BV}$ in reducing the number of acute exacerbations of bronchiectasis in Chinese patients. OM- 
Table 4 Summary of secondary efficacious end-points

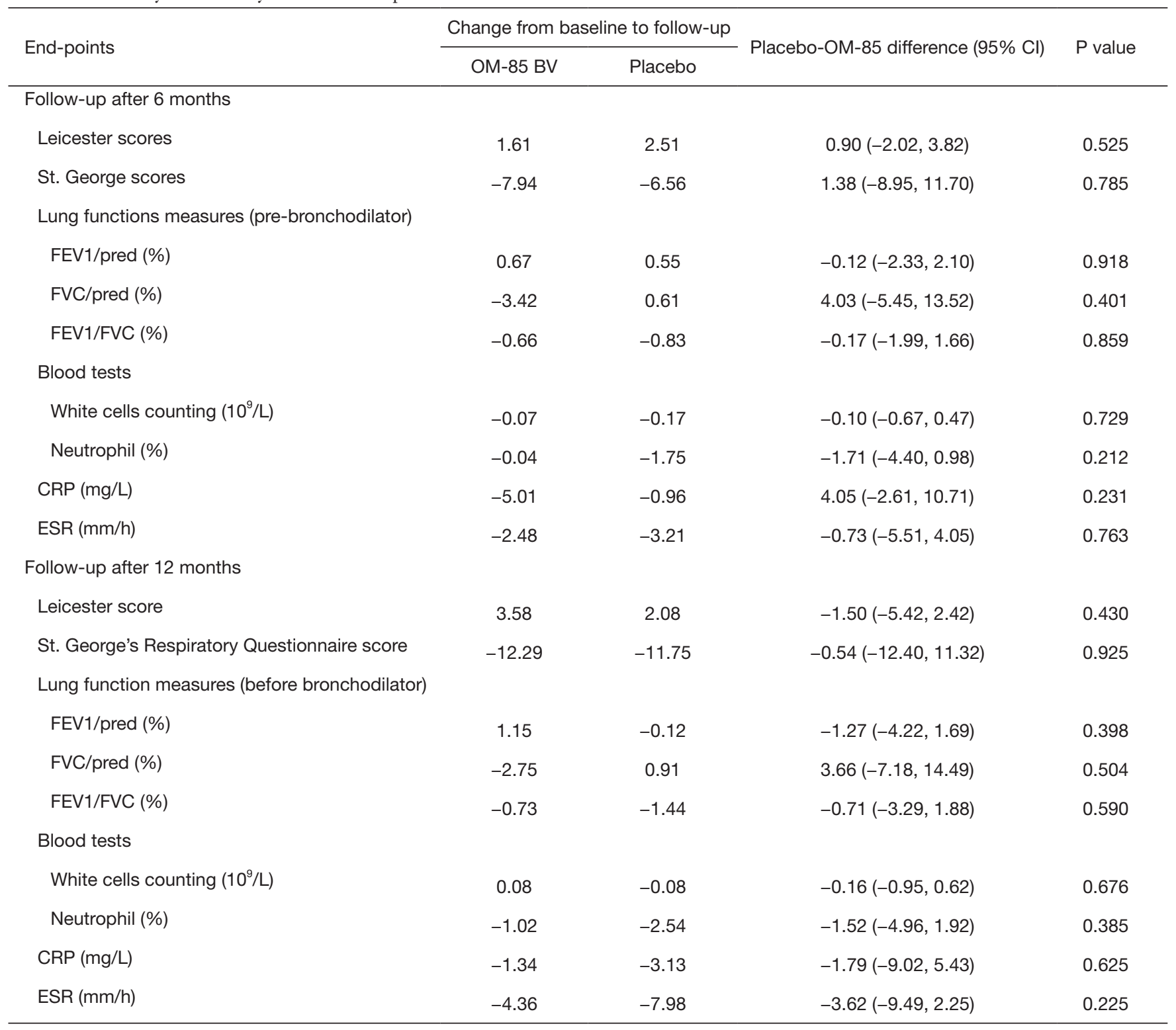

CRP, C-reactive protein; ESR, erythrocyte sedimentation rate; FEV1/pred, forced expiratory volume in first second/predicated; FVC/pred, forced vital capacity/predicated; $\mathrm{Cl}$, confidence interval.

Table 5 Comparison of duration of acute exacerbations and antibiotics use

\begin{tabular}{lccc}
\hline Variable & OM-85 BV $(\mathrm{n}=116)$ & Placebo $(\mathrm{n}=117)$ & $\mathrm{P}$ value \\
\hline $\mathrm{AE}$ (days) & $8.84 \pm 4.44$ & $9.11 \pm 4.15$ & 0.723 \\
Antibiotics use (days) & $9.19 \pm 4.48$ & $9.20 \pm 3.82$ & 0.989 \\
\hline
\end{tabular}

$\mathrm{AE}$, acute exacerbation. 
Table 6 Summary of adverse events

\begin{tabular}{lccc}
\hline Adverse events categorized by organ & OM-85 $(\mathrm{n}=99), \mathrm{n}(\%)$ & Placebo $(\mathrm{n}=97), \mathrm{n}(\%)$ & Total $(\mathrm{n}=196), \mathrm{n}(\%)$ \\
\hline Any adverse events & $7(7.07)$ & $9(9.28)$ & $16(8.16)$ \\
Severe adverse events & $2(1.01)$ & $1(1.03)$ & $3(1.53)$ \\
Palpitation & $0(0)$ & $1(1.03)$ & $1(0.51)$ \\
Nausea & 0 & $1(0.85)$ & $1(0.43)$ \\
Abdominal discomfort & $1(1.01)$ & $2(2.06)$ & $3(1.53)$ \\
Common cold & $3(3.03)$ & $4(4.12)$ & $7(3.57)$ \\
Arthralgia & 0 & $1(1.03)$ & $1(0.51)$ \\
Miscellaneous & $3(3.03)$ & $0(0)$ & $3(1.53)$ \\
\hline
\end{tabular}

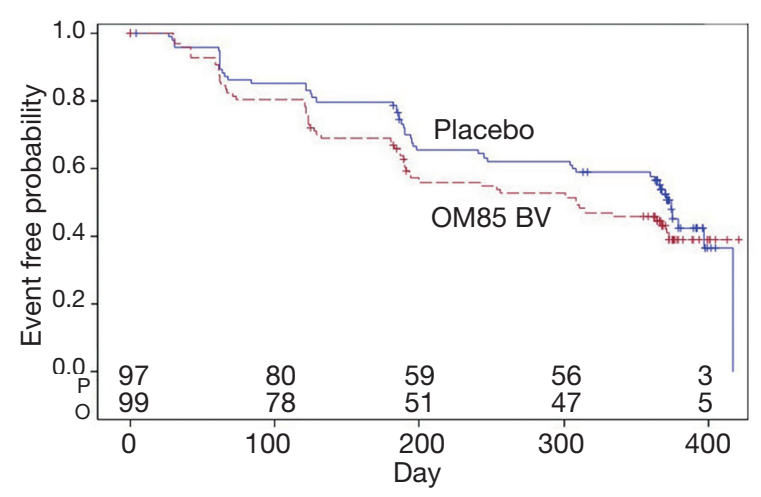

Figure 3 Kaplan-Meier curve of the time to first pulmonary exacerbation of any severity in the modified intention-to-treat (mITT) population. P: placebo; O: OM-85 BV.

85 BV did not demonstrate any clinically relevant beneficial effects on cumulative number of acute exacerbations, the time to first exacerbation, lung function improvement, cough symptoms and quality of life using the Leicester and the SGRQ scores (FAS analysis). Similar observations were recorded in the per protocol analysis.

Although the present study failed to demonstrate a benefit for OM-85 in bronchiectasis exacerbations, it is nonetheless a valid negative study.

It may be speculated multiple pathways involving in pulmonary exacerbations of bronchiectasis.

We propose several possibilities to explain our negative results. First this relates to the mechanism of action for OM-85 BV through direct activation of lung macrophages by OM-85 $\mathrm{BV}$ in the respiratory tract $(29,30)$. Bronchiectasis is a chronic suppurative lung disease characterized by neutrophil-predominant airways inflammation (31). Data from animal experiment showed that OM-85 BV had little effect on neutrophils evidenced by a failure to bacterial clearance in Klebsiella pneumoniaeinduced pneumonia, thus, no protective effect in a recurrent infection with this bacterium (32). This might suggest that alveolar macrophage activation by systemic administration with OM-85 BV is not sufficient for the protection for neutrophilic inflammation in respiratory tract bacterial infection (32). Similarly, one large randomized placebocontrolled trial performed in COPD patients showed that OM-85 didn't reduce the total number of exacerbations compared with placebo (28). OM-85 BV was shown to be more efficacious for COPD patients with a history of current or ex-smokers in decreasing exacerbations (19). Our present individual study included a small number of current smokers, thus, we were unable to perform the meaningful subgroup analysis.

The nature of disease heterogeneity may account for the difficulty in achieving endpoints in clinical trials (33). This clinical trial failed to demonstrate the additional benefit of OM-85 BV to prevent acute exacerbation of bronchiectasis on basis of the regular therapies, on the hand, reflecting the complex and heterogeneity of this disorder. It may be that recognizing the treatable traits of bronchiectasis would be of clinical relevance (34), therefore, the study with the objective aiming to identify the subgroups of patients (i.e., those having at least 3 exacerbations in the previous year) in whom OM-85 has beneficial effect is needed.

One potential reason why OM-85 BV did not lead to a significant decrease in bronchiectasis exacerbations is that it does not include bacterial lysates, Pseudomonas aeruginosa, which was the most common pathogen in Chinese patients with bronchiectasis $(1,2)$. In contrast, OM-85 BV has been shown to decrease COPD exacerbations, where the most 
common bacterial etiologies include those lysates included in this oral formulation. The similarly modest decrease in the number of exacerbation was observed in both groups. This might underline the importance of the increased awareness of the disease and daily care for bronchiectasis patients. We have to point out that existence of a ceiling effect might be an alternative explanation for the negative results of OM-85 BV in this trial. It was evidenced by the high rate of prescriptions for concomitant respiratory medications at the time recruitment and throughout the entire study period. However, we can't exclude the possibility of underreporting of events that might attribute to the negative result.

The strength of this current trial was the study design, which to a larger extent, replicated the methods previously adopted in trials with the objective of decreasing acute exacerbation of COPD and bronchiectasis (12-14,19,28). This trial lasted for 12 months, a period long enough to avoid bias due to seasonal variability in exacerbation frequency and time interval required for the typical patient to experience an exacerbation. These indicated that the trial design was sufficient to demonstrate the preventive property of OM-85 in bacterial exacerbations of bronchiectasis.

There were limitations to this study design. One might be related to the inclusion of ten medical centers in geographically different regions across China, leading to assessment of the potentially heterogeneous populations. The method for the assessment of acute events was pragmatic, information reported from both self-report by the patients and medical records. However, the blinded nature of this evaluation and the study size made us believe that these observations were not likely to be invalid. Demographic differences between the study drug and placebo groups at baseline were thought likely to affect the primary outcome of the study. It has been shown that more patients $(64.6 \%)$ had a longer duration of disease when recruiting in OM-85 BV group than those in placebo group in this current trial. In addition, there was heterogeneity in the number of acute exacerbations at baseline exampled by recruitment of patients having at least 1 exacerbation. In spite of the modest size of enrolled subjects, the iPROBE study was a large bronchiectasis trial compared with other studies of reducing bronchiectasis exacerbations by macrolides (12-14).

We did not find any significant difference in SGRQ and LCQ scorings between two groups during the trial. This is likely due to the fact that scorings evaluated at designated times distant from the exacerbation might insensitive to the effect of the drug on decreasing pulmonary exacerbations. Also, there was similar change in lung function measurements in both groups after 1-year trial. These results are consistent with those observed in the trial of reducing exacerbations of COPD by OM-85 BV (28).

$\mathrm{OM}-85 \mathrm{BV}$ has been widely used to prevent recurrent respiratory infections and has demonstrated a favorable AEs profile. Our trial confirms this safety profile. The majority of the documented AEs shown in Table 5 were not severe, self-limited, and resolved fully. The total number and the distribution of events were similar in the two groups and compatible with what has been observed previously in COPD exacerbation trials $(18,20,28)$.

In summary, the iPROBE study was not able to demonstrate the benefit for OM-85 BV, on the basis of regular treatment, in the prevention of acute exacerbation of bronchiectasis in Chinese patients. However, our negative results don't necessarily invalidate the strategy of enhancing the host body immunity against airway infections. Therefore, a logic stratified approach to treatment will be required in future. In particular, longer-term efficacy of OM-85 BV with the treatment period of more than 1 year in carefully phenotyped patients may provide additional insight into the role of this therapy for bronchiectasis.

\section{Acknowledgments}

The authors would like to thank Profs. Xiang Gao, Guangliang Shan, Huijing He, and Dr. Huiru Ren for the help in statistical analysis and important suggestions. We thank Drs. Ping Chen, Jinna Li, Zhuozhe Li, Kebing Cheng, Dongqing Zhang, Hong Zhu, Chun Chang, Xuefen Wang, and Hequan Li for the help in screening and recruiting the patients. The authors would like to thank all subjects for agreeing to participate in this study.

Funding: This work was in part supported by ViforPharm and UCB pharmaceuticals.

\section{Footnote}

Data Sharing Statement: Available at http://dx.doi. org/10.21037/jtd-20-1662

Conflicts of Interest: All authors have completed the ICMJE uniform disclosure form (available at http://dx.doi. org/10.21037/jtd-20-1662). The authors have no conflicts of interest to declare. 
Ethical Statement: The authors are accountable for all aspects of the work in ensuring that questions related to the accuracy or integrity of any part of the work are appropriately investigated and resolved. The study was conducted in accordance with the Declaration of Helsinki (as revised in 2013) and the Harmonized Tripartite Guideline for GCP from the International Conference on Harmonization. The study was reviewed and approved by the Human Research Ethics Board at Peking Union Medical College Hospital (REB number S-474). All patients enrolled completed the informed consent form.

Open Access Statement: This is an Open Access article distributed in accordance with the Creative Commons Attribution-NonCommercial-NoDerivs 4.0 International License (CC BY-NC-ND 4.0), which permits the noncommercial replication and distribution of the article with the strict proviso that no changes or edits are made and the original work is properly cited (including links to both the formal publication through the relevant DOI and the license). See: https://creativecommons.org/licenses/by-nc-nd/4.0/.

\section{References}

1. Li Z, Li JR, Gao JM. Clinical evaluation of 136 inpatients with bronchiectasis in Peking Union Medical College Hospital. Zhongguo Yi Xue Ke Xue Yuan Xue Bao 2014;36:61-7.

2. Lin JL, Xu JF, Qu JM. Bronchiectasis in China. Ann Am Thorac Soc 2016;13:609-16.

3. Quint JK, Millett ER, Joshi M, et al. Changes in the incidence, prevalence and mortality of bronchiectasis in the UK from 2004 to 2013: a population-based cohort study. Eur Respir J 2016;47:186-93.

4. Ringshausen FC, de Roux A, Diel R, et al. Bronchiectasis in Germany: a population-based estimation of disease prevalence. Eur Respir J;46:1805-7.

5. Chalmers JD, Aliberti S, Blasi F. Management of bronchiectasis in adults. Eur Respir J 2015;45:1446-62.

6. Whitters D, Stockley R. Immunity and bacterial colonisation in bronchiectasis. Thorax 2012;67:1006-13.

7. Chalmers JD, Hill AT. Mechanisms of immune dysfunction and bacterial persistence in non-cystic fibrosis bronchiectasis. Mol Immunol 2013;55:27-34.

8. Angrill J, Agusti C, de Celis R, et al. Bacterial colonisation in patients with bronchiectasis: microbiological pattern and risk factors. Thorax 2002;57:15-9.

9. Hill AT, Haworth CS, Aliberti S, et al. Pulmonary exacerbation in adults with bronchiectasis: a consensus definition for clinical research. Eur Respir J 2017;49:1700051.

10. Polverino E, Goeminne PC, McDonnell MJ, et al. European Respiratory Society guidelines for the management of adult bronchiectasis. Eur Respir J 2017;50:1700629.

11. Altenburg J, de Graaff CS, van der Werf TS, et al. Immunomodulatory effects of macrolide antibiotics - part 2: advantages and disadvantages of long-term, low-dose macrolide therapy. Respiration 2011;81:75-87.

12. Wong C, Jayaram L, Karalus N, et al. Azithromycin for prevention of exacerbations in non-cystic fibrosis bronchiectasis (EMBRACE): a randomised, double-blind, placebo-controlled trial. Lancet 2012;380:660-7.

13. Altenburg J, de Graaff CS, Stienstra Y, et al. Effect of azithromycin maintenance treatment on infectious exacerbations among patients with non-cystic fibrosis bronchiectasis: the BAT randomized controlled trial. JAMA 2013;309:1251-9.

14. Serisier DJ, Martin ML, McGuckin MA, et al. Effect of long-term, low-dose erythromycin on pulmonary exacerbations among patients with non-cystic fibrosis bronchiectasis: the BLESS randomized controlled trial. JAMA 2013;309:1260-7.

15. Chalmers JD, Boersma W, Lonergan M, et al. Long-term macrolide antibiotics for the treatment of bronchiectasis in adults: an individual participant data meta-analysis. Lancet Respir Med 2019;7:845-54.

16. Rogers GB, Bruce KD, Martin ML, et al. The effect of long-term macrolide treatment on respiratory microbiota composition in non-cystic fibrosis bronchiectasis: an analysis from the randomised, double-blind, placebo-controlled BLESS trial. Lancet Respir Med 2014;2:988-96.

17. Emmerich B, Pachmann K, Milatovic D, et al. Influence of OM-85 BV on different humoral and cellular immune defense mechanisms of the respiratory tract. Respiration 1992;59 Suppl 3:19-23.

18. Orcel B, Delclaux B, Baud M, et al. Oral immunization with bacterial extracts for protection against acute bronchitis in elderly institutionalized patients with chronic bronchitis. Eur Respir J 1994;7:446-52.

19. Soler M, Mutterlein R, Cozma G, et al. Double-blind study of OM-85 in patients with chronic bronchitis or mild chronic obstructive pulmonary disease. Respiration 2007;74:26-32.

20. Tang H, Fang Z, Saborio GP, et al. Efficacy and Safety 
of OM-85 in Patients with Chronic Bronchitis and/ or Chronic Obstructive Pulmonary Disease. Lung 2015;193:513-9.

21. Pasteur MC, Bilton D, Hill AT, et al. British Thoracic Society guideline for non-CF bronchiectasis. Thorax 2010;65 Suppl 1:i1-58.

22. Gao J, Gao X, Kong L. To investigate the prevention of OM-85 on bronchiectasis exacerbations (iPROBE) in Chinese patients: study protocol for a randomized controlled trial. Trials 2014;15:150.

23. Anthonisen NR, Manfreda J, Warren CP, et al. Antibiotic therapy in exacerbations of chronic obstructive pulmonary disease. Ann Intern Med 1987;106:196-204.

24. Wilson CB, Jones PW, O'Leary CJ, et al. Validation of the St. George's Respiratory Questionnaire in bronchiectasis. Am J Respir Crit Care Med 1997;156:536-41.

25. Murray MP, Turnbull K, MacQuarrie S, et al. Validation of the Leicester Cough Questionnaire in non-cystic fibrosis bronchiectasis. Eur Respir J 2009;34:125-31.

26. Little RJ, D'Agostino R, Cohen ML, et al. The prevention and treatment of missing data in clinical trials. $\mathrm{N}$ Engl J Med 2012;367:1355-60.

27. Lusuardi M, Capelli A, Carli S, et al. Local airways immune modifications induced by oral bacterial extracts in chronic bronchitis. Chest 1993;103:1783-91.

28. Collet JP, Shapiro P, Ernst P, et al. Effects of an

Cite this article as: Gao J, Li L, Jiang N, Liao Y, Kong L, Song Y, Xu J, Cao J, Li Y, Que C, Pleasants RA. Effect of OM$85 \mathrm{BV}$ on reducing bronchiectasis exacerbation in Chinese patients: the iPROBE study. J Thorac Dis 2021;13(3):1641-1651. doi: $10.21037 /$ jtd-20-1662 immunostimulating agent on acute exacerbations and hospitalizations in patients with chronic obstructive pulmonary disease. The PARI-IS Study Steering Committee and Research Group. Prevention of Acute Respiratory Infection by an Immunostimulant. Am J Respir Crit Care Med 1997;156:1719-24.

29. Mauel J, Van Pham T, Kreis B, et al. Stimulation by a bacterial extract (Broncho-Vaxom) of the metabolic and functional activities of murine macrophages. Int J Immunopharmacol 1989;11:637-45.

30. Mauel J. Macrophage activation by OM-85 BV. Respiration 1992;59 Suppl 3:14-8.

31. Bedi P, Davidson DJ, McHugh BJ, et al. Blood Neutrophils Are Reprogrammed in Bronchiectasis. Am J Respir Crit Care Med 2018;198:880-90.

32. Broug-Holub E, Kraal G. In vivo study on the immunomodulating effects of OM-85 BV on survival, inflammatory cell recruitment and bacterial clearance in Klebsiella pneumonia. Int J Immunopharmacol 1997;19:559-64.

33. Flume PA, Chalmers JD, Olivier KN. Advances in bronchiectasis: endotyping, genetics, microbiome, and disease heterogeneity. Lancet 2018;392:880-90.

34. Boaventura R, Sibila O, Agusti A, et al. Treatable traits in bronchiectasis. Eur Respir J 2018;52:1801269. 